Philosophie ANTIQUE

\section{Philosophie antique}

Problèmes, Renaissances, Usages

15 | 2015

Questions sur le scepticisme pyrrhonien

\title{
Dimitri EL MURR, Savoir et gouverner, Essai sur la science politique platonicienne
}

\author{
Fulcran Teisserenc
}

\section{(2) OpenEdition}

\section{Journals}

Édition électronique

URL : https://journals.openedition.org/philosant/453

DOI : 10.4000/philosant.453

ISSN : 2648-2789

\section{Éditeur}

Éditions Vrin

\section{Édition imprimée}

Date de publication : 24 novembre 2015

Pagination : 262-265

ISBN : 978-2-7574-1141-4

ISSN : $1634-4561$

\section{Référence électronique}

Fulcran Teisserenc, «Dimitri el muRr, Savoir et gouverner, Essai sur la science politique platonicienne », Philosophie antique [En ligne], 15 | 2015, mis en ligne le 01 novembre 2018, consulté le 04 décembre 2022. URL : http://journals.openedition.org/philosant/453 ; DOI : https://doi.org/10.4000/philosant. 453

\section{(c) (i) (3)}

Creative Commons - Attribution - Pas d'Utilisation Commerciale - Pas de Modification 4.0 International - CC BY-NC-ND 4.0

https://creativecommons.org/licenses/by-nc-nd/4.0/ 
semble très précieuse sur un sujet central, liant psychologie et éthique platoniciennes.

Charlotte Murgier

Université Paris Est Créteil Val de Marne

Dimitri EL MURR, Savoir et gouverner, Essai sur la science politique platonicienne, Paris, Librairie philosophique J. Vrin, 2014 (Tradition de la pensée classique), 336 p. ISBN 978-2-7116-2586-4

Dans cet ouvrage, Dimitri El Murr reprend la question posée par Platon - et, depuis, à l'horizon de toutes nos institutions politiques -, des rapports entre savoir et pouvoir, en réexaminant la portée et les analyses du Politique, contribution majeure de la réflexion platonicienne à ce problème. Que le savoir soit en lui-même un pouvoir, c'est ce que Socrate soutenait dans le Protagoras et le Gorgias, mais ce pouvoir restait celui de l'âme sur elle-même et sa conduite. À partir de la République, l'enjeu changea en partie : il glissa vers le bénéfice que la cité peut retirer de son administration par des philosophes. Toutefois, le type de compétence que ces derniers sont appelés à mettre en œuvre dans l'exercice de leurs responsabilités n'était encore guère examiné.

C'est cette lacune que le Politique vient combler. L'auteur s'emploie à le montrer en discutant trois points controversés: 1) les liens entre dialectique et politique ; 2) la signification des divisions et du grand mythe cosmologique ; 3) le paradigme du tissage et son application. Sont ainsi mises en évidence la continuité du parcours, la pertinence de certaines distinctions fondamentales et la scientificité même de la vraie politique.

I. Après avoir examiné la réception du Politique, l'A. s'interroge sur la congruence d'une réflexion sur la démarche philosophique et d'une enquête sur le politique. Les deux objectifs sont compatibles : l'inventaire soigné des différences spécifiques poursuivi pour lui-même permet aussi d'insérer la science politique dans un réseau de déterminations qui en précise au plus juste la nature.

Autre question de méthode, celle des paradigmes. L'exemple de l'apprentissage de la lecture illustre leur fonction d'exercice et de découverte. Mais ce point de méthode éclaire le rapport du dialogue à son contexte : si le sophiste est le simulacre du philosophe, le politique en serait la copie, et l'enquête sur la politique peut être vue comme le paradigme de celle sur la dialectique. L'A. souligne aussi que le paradigme de substitution, que ce soit celui du pêcheur dans le Sophiste ou du tissage dans le Politique, sert également à désamorcer préjugé et surestimation, qui brouillent l'appréciation correcte de l'objet cible quand il est étudié directement et non par transposition.

Qu'en est-il maintenant de l'unité structurelle du dialogue ? L'A. met en exergue la continuité de la diérèse tout le long du dialogue, dichotomique d'abord, polytomique ensuite; mais cette modification tient compte des leçons des deux grandes interruptions apparentes - le mythe, l'étude du tissage -, lesquelles ne sont 
à chaque fois qu'une manière de réorienter et de corriger la division en cours. Et les incises méthodologiques sont elles-mêmes commandées par la prise de conscience de l'inadéquation du paradigme en usage. Ainsi les premières divisions s'avèrent fautives parce qu'elles se sont appuyées sur un modèle pastoral implicite, limité à sa dimension nourricière - première erreur que corrige le mythe en réintroduisant la dimension du soin. Mais même ainsi amendé, il reste trop large et ne permet pas de préciser suffisamment le portrait du roi - seconde erreur corrigée par la comparaison avec le tissage.

II. Les premiers partages opérés par l'Étranger sont fondamentaux pour la définition du politique.

Tout d'abord, l'affirmation inaugurale que le politique compte au nombre de ceux qui savent. Ce qui revient bien sûr à faire de la politique une science (et non une opinion droite par exemple). Une décision est donc prise, justifiée par le développement de la définition - c'est ainsi que l'A. répond à l'étonnement stupéfait en ce point de C. Castoriadis. Mais ne doit-on pas reconnaître que cette décision fixe un cap dont jamais les protagonistes du dialogue ne s'écarteront ? Il est d'autres textes platoniciens où la question fut formulée de façon beaucoup plus ouverte, dans le Ménon par exemple (92b).

La deuxième difficulté que discute l'A. à propos de ces premières divisions concerne deux autres affirmations, l'une, associant en une même unité la compétence politique et celle du maître du domaine et du chef des esclaves, l'autre, faisant de la science politique une science « cognitive » et non point pratique. Pour l'A., l'introduction de la figure du conseiller, grâce à laquelle peuvent être distingués le véritable politique, possesseur de la science adéquate, et le roi de fait, qui n’a pour lui que le pouvoir exécutif, est destinée à jouer le rôle de proposition médiatrice entre les deux propositions précédentes. D’une part, le conseiller, détaché des contraintes immédiates de l'action, fait ressortir la nature profondément épistémique de l'art politique, qui se définit par sa compétence à déterminer scientifiquement ce qu'il faut faire (et non point à veiller à son exécution), tandis que sa fonction non officielle en fait une figure potentiellement privée, à l'image du chef de maison ou du maître des esclaves. Mais, d'autre part, le regroupement dans une même catégorie du despotes et du politikos est destiné à rendre sensible l'autorité qui découle de la seule possession de la science politique, alors que cette autorité aurait pu être contestée du fait précisément qu'elle soit logée in fine chez le conseiller et non chez celui qui est investi publiquement du pouvoir : le maitre de maison exerce dans son domaine une autorité qui n'a pas besoin d'une reconnaissance politique expresse (élection, couronnement, allégeance...).

Quant au rapport plus particulier d'Aristote à Platon, l'A. tente de montrer que ce dernier ne tombe pas sous le coup de la critique de son élève, qui conteste que la différence entre la maison et la cité soit seulement une différence quantitative. En effet, Platon mettrait pour sa part l'accent sur le fait qu'il s'agirait dans tous les cas d'une science du commandement impliquant celle du bien - des es- 
claves, des femmes et des enfants, des citoyens; le savoir du bien unifierait donc gouvernement domestique et gouvernement politique.

La suite des premières divisions du dialogue vise à identifier l'animal objet du soin politique. L'A. s'attache à montrer que les divisions n'ont pas de but taxinomique et qu'elles procèdent à une réduction provocatrice de l'être pris en charge par la politique, ironiquement défini selon des critères exclusivement biologiques, tenant à l'anatomie, à la reproduction, à la locomotion ou encore à l'habitat. Voilà qui fait délibérément l'économie de l'homme rationnel et relationnel : le bipède sans plume est cet « animal dont la nature politique est nécessairement acquise et construite sous l'effet de la science » (p. 140).

Le mythe est un texte fort complexe, qui interrompt le cours de la division car, explique l'A, celle-ci ne peut rendre raison de l'histoire et de la différence des temps. L'A. défend une lecture dualiste, assez traditionnelle, selon laquelle le monde connaîtrait l'alternance perpétuelle de deux cycles, correspondant à l'âge de Cronos et à celui de Zeus. La thèse principale de l'A. est que l'âge de Cronos est un âge apolitique, tandis que l'âge de Zeus est un âge prépolitique. Les hommes sous Cronos vivent sans liens sociaux, ni même familiaux, sans cité et sans constitution, sans technique non plus et sans travail. Faute d'une participation des humains à leur propre bonheur, entièrement réglé de l'extérieur, celui-ci n'est la manifestation d'aucune vertu, tant de l'intelligence que du caractère. Il possède par conséquent une perfection moindre que celle susceptible d'être obtenue à l'âge de Zeus (p. 182). Puisque, comme le remarque l'A., il n'y a pas de possibilité de devenir meilleur sous Cronos, la science politique à définir se distingue nécessairement du pastorat divin ; car elle se propose justement comme finalité un tel devenir, lequel est sans parallèle dans l'époque précédente.

Quant à la description par l'Étranger de la vie que mènent les hommes sous Zeus, elle ne correspond que partiellement au mythe de Protagoras : s'il est fait état des dons divins, ceux-ci se limitent à la sphère technique, et il n'est rien dit dans le mythe du Politique qui fasse écho à la distribution universelle de l'aidos et de la dike, ces dispositions morales qui forment selon le sophiste la base de la compétence démocratique. Ce silence correspond à la place, encore vacante, que viendra occuper la science politique.

III. La riche et subtile analyse du tissage et de la science politique occupe la dernière partie de l'ouvrage, à laquelle je ne peux rendre tout à fait justice ici.

Je me limiterai à un point que je crois décisif. Faut-il considérer, comme le suggère l'A. (p. 219), que la subordination de la rhétorique, de la stratégie et de l'art judiciaire à un art épitactique (la politique) suffise à garantir leur scientificité ? Socrate le contestait déjà dans le Gorgias en dénonçant la routine, l'empeiria, de la rhétorique $(463 \mathrm{~b}-\mathrm{c})$. On peut certes imaginer une rhétorique plus dialectique, comme dans le Phèdre (271c-272a), fondée sur une double connaissance diérétique, se rapportant d'un côté aux types d'âmes, et de l'autre aux formes de discours qui leur sont appropriées. Mais se pose le problème de l'action concrète : 
comment la rhétorique s'y prendra-t-elle pour identifier avec sûreté la catégorie psychique dont relève tel ou tel citoyen qui doit être persuadé par une forme déterminée de discours ? Difficulté qui revient d'une autre manière quand il faudra discerner empiriquement les tempéraments (les modérés ou les ardents) et éliminer les enfants non éducables (308e-309b). Le politique tisse un matériau qu'il a luimême choisi ( $c f$. p. 274 in fine) : quel savoir dirigera avec autorité et rigueur son doigt sélectif ? Il est introuvable dans le cadre de l'épistémologie générale de Platon. L'Étranger le dit à sa manière, sur un autre plan : la politique est aussi connaissance du kairos, du moment opportun qui permettra de dire si oui ou non il faut recourir à la rhétorique, à la stratégie ou à l'art judiciaire, autrement dit, s'il faut (ou non) persuader, faire la guerre, lancer un procès (305d). Ce kairos est éminemment changeant, il n'obéit à aucune loi, il n'a pas de critère assuré - il est cette fenêtre qu'ouvre provisoirement le devenir à une action susceptible d'orienter son cours dans une certaine direction. Mais, du kairos (comme d'ailleurs du choix des caractères), il n'y a pas de science, car il n'y a de science que de ce qui est toujours identique à soi, de ce qui, précisément, est hors du devenir.

Peut-on réduire la contradiction entre l'épistémologie générale de Platon et sa conception particulière de la science politique dans notre dialogue ? La question reste en suspens. À moins, peut-être, que cette science ne soit seulement celle des bonnes intentions : viser le bien, préférer la limite à l'illimité, introduire la juste mesure, ce qui passerait par l'institution d'une orthe doxa et l'assortiment réglé des tempéraments contraires. Admettons. Mais en l'absence d'un modus operandi déterminé, il s'agit là d'une science terriblement formelle et vague : l'exigence de détermination et de mesure manque à son tour de détermination et de mesure - et s'avère incapable d'asseoir la légitimité du politique.

Quoi qu'il en soit de cette difficulté sans doute insoluble, l'ouvrage de Dimitri El Murr demeure pour qui veut comprendre le Politique, et plus largement la pensée platonicienne, une référence obligatoire, tant par la finesse de ses analyses et la clarté de son écriture que par les questions qu'il invite à poser.

Fulcran TEISSERENC

GRAMATA, Université Paris I Panthéon-Sorbonne

Jakob L. FINK (éd.), The Development of Dialectic from Plato to Aristotle, VII355p., Cambridge-New York, Cambridge University Press, 2012, ISBN 978-1107-01222-6

Actes d'un colloque qui s'est tenu à Copenhague en 2007, l'ouvrage veut combler un manque : traiter du développement de la dialectique de Platon à Aristote, non pas en se focalisant sur la méthode et l'ontologie, mais en se fondant sur le cadre du débat dialectique. Selon J. Fink (p. 2), la dialectique ici signifie d'abord 